\title{
PROSPECTION GEOPHYSIQUE POUR LA RECHERCHE DES EAUX SOUTERRAINES A SILIDARA (CONCESSION DE CBG)
}

\author{
Kourouma Mory ${ }^{1}$, Keita Daouda ${ }^{1}$, Viktorov Gleb ${ }^{2}$ \\ ISMGB, Guinée \\ Toumnyne, Guinée
}

Résumé: la Compagnie des Bauxites de Guinée, dans le cadre d'approvisionnement de ses sites miniers à SILIDARA à Sangaredi (Concession CBG) en eau potable, a réalisé des forages d'hydraulique. La société de prospection TOUMNYNE S.A.R.L. a été chargée d'exécuter ce projet. Cette société a effectué, à cet effet, la prospection géophysique des eaux souterraines avant l'implantation des points d'eau sur ces sites. La méthode géophysique utilisée par TOUMNYNE S.A.R.L. est la méthode magnétique avec filtrage des résultats. La prospection magnétique a été faite suivant sept (07) profils à l'aide du magnétomètre à protons de marque MMP-203MS (production de la Russie).

Le traitement des résultats ont permis de mettre en évidence trois zones de fissures accompagnés de broyage (03 zones de broyage) disposées de façon linéaire. La valeur maximale des anomalies magnétiques a été fixée à $32 n T$.

Mots clés: sites miniers, SILIDARA, prospection magnétique, anomalies magnétiques, fissures, zones de broyage.

Abstract: the Bauxite Company of Guinea, in the procurement framework of its mining sites in SILIDARA Sangaredi (CBG Concession) in drinking water, has made hydraulic drilling. The exploration company TOUMNYNE S.A.R.L. was responsible for executing the project. This company had, for this purpose, the geophysical groundwater before implantation of water points on these sites. The geophysical method used by TOUMNYNE s.a.r.l is the magnetic method with filtering results. The magnetic survey was made following seven (07) profiles using the branded proton magnetometer MMP- 203MS (Russian production).

The treatment results helped to highlight three areas of cracks accompanied by grinding (milling zones 03) linearly arranged. The maximum value of the magnetic anomalies was set at $32 \mathrm{nT}$.

Key words: mining sites, SILIDARA, magnetic prospecting, magnetic anomalies, fault, broyage areas.

\section{Introduction}

Les travaux de prospection magnétique ont été effectués dans le cadre de solution du problème de ravitaillement en eau potable de sites miniers au NORD de la région de Boké.

Avant la prospection magnétique, on disposait des données suivantes : 
La coupe géologique générale de la zone d'étude est caractérisée par une couverture sédimentaire récente d'épaisseur prédominante de 5 - 10 mètres, composée de latérites et d'argiles.

Par l'intermédiaire de 3 à 5 mètres de roche mère altérée, elle repose soit sur la roche cristalline saine représentée dans cette région essentiellement par la dolérite du Mésozoïque, soit par des aleur?lites/argilites dévoniennes.

Les dolérites comme les aleurolites sont affectées de plusieurs failles, fractures, zones broyées et affaiblies.

Concernant les conditions hydrogéologiques, on peut dire avec une grande probabilité, que le seul réservoir capable d'encaisser des quantités d'eau considérables est celui des fissures, des fractures avec zones broyées etc. dans les aleurolites et dolérites.

La recherche des sources des eaux sera donc orientée vers des fractures majeures dans les roches mères à prédominance doléritique.

La tache essentielle qu'on ait posée devant la prospection magnétique est la recherche de failles et de zones de broyage comme des structures perspectives pour implanter des forages d'eaux.

\section{Matertiels et methodes}

\subsection{Equipement}

Pour réaliser les travaux de prospection magnétique sur le terrain on a organisé une équipe de travail contenant un opérateur, un aide opérateur et un secrétaire. Cette équipe a utilisé le magnétomètre moderne à proton de marque MMP-203MS (production de la Russie) qui donne la possibilité de mesurer le module du vecteur total du champ magnétique terrestre dans le diapason entre 20000 et 100 000nT. La valeur minimale mesurée égale à $1 \mathrm{nT}$.

\subsection{Maille d'observation}

On a choisi, d'après les objectifs des travaux, la maille d'observation de $10 \times 10 \mathrm{~m}$ : $10 \mathrm{~m}$ est la distance entre les profils et 10 $\mathrm{m}$ est la distance entre les points d'observation. La maille $10 \times 10 \mathrm{~m}$ à été implantée par la station totale TRIMBLE M3.

\subsection{Contrôle des mesures}

Pour effectuer le contrôle de mesure du champ magnétique on a répété des observations aux certaines nombre des points sur les profils d'observation. Les mesures de contrôle nous ont permis de 
calculer l'erreur moyenne d'une lecture isolée d'après la formule suivante

$$
\varepsilon=\sqrt{\frac{\sum_{i=1}^{N}\left(X_{i}^{\prime}-X_{i}\right)^{2}}{2 N}}
$$

$X_{i}^{\prime}$ - lecture ordinaire;

$X_{i}$ - lecture de contrôle ;

$N$ - nombre des points réitérés.

\section{Resultats et discutions}

3.1. Etude de variations du champ magnétique terrestre

Pour étudier le caractère des variations du champ magnétique terrestre on a choisi une station de mesure des variations diurnes du champ magnétique sur laquelle on a mesuré le champ magnétique toutes les 5 minutes à partir de $10 \mathrm{~h}$ et jusqu'à fin de travail. Le caractère de variations du champ magnétique terrestre est présenté sur la fig. 1. On peut voir que d'une part, le champ magnétique terrestre monte du matin vers le midi, et puis redescend dans l'après midi ; et d'autre part, les variations diurnes du champ magnétique portent le caractère monotone linéaire. L'amplitude maximale des variations diurnes ne dépasse pas 35 nT. Pour éliminer l'influence des variations diurnes du champ magnétique il a été enregistré au niveau de chaque piquet le temps de mesure ce qui a permis d'introduire les corrections nécessaires.

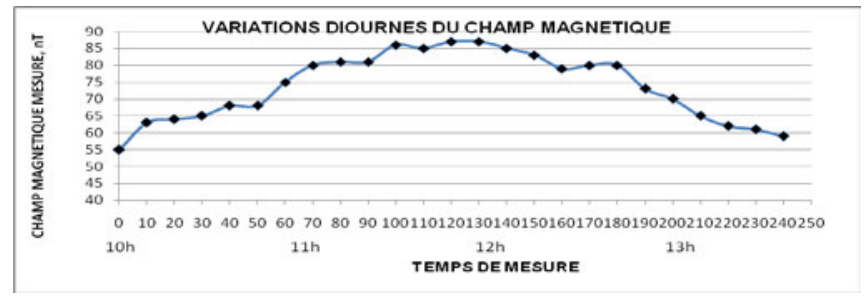

Fig. 1. Variations diurnes du champ magnétique des sites

\subsection{Résultats de mesures du champ magnétique}

La valeur moyenne générale du champ magnétique mesuré sur les sites est égale à $32101 n T$, ce qui est très proche du champ 
magnétique normal de la Terre dans cette région (33654 nT), calculé d'après la formule suivante

$$
T=\frac{\mu_{0}}{4 \pi} \frac{M}{R^{3}} \sqrt{1+3 \sin ^{2} \varphi_{m}}
$$

$R$ - rayon de la Terre $(R=6371 \mathrm{~km})$;

$M$ - moment magnétique de la Terre $\left(8,3 \cdot 10^{22} \mathrm{~A} \mathrm{~m}^{2}\right)$;

$\mu_{0}$ - constante magnétique ( $\left.4 \pi \cdot 10^{-7} \mathrm{~m}^{2} \mathrm{~kg} / \mathrm{sec}^{2} \mathrm{~A}^{2}\right)$;

$\varphi_{m}$ - latitude du site de travail (dans notre cas $\varphi_{m}=10,5^{\circ}$ )

Sur la fig. 2 sont représentés les graphiques du champ magné?iques corrigés sur les variations diurnes du champ magnétique terrestre et le champ magnétique normal.

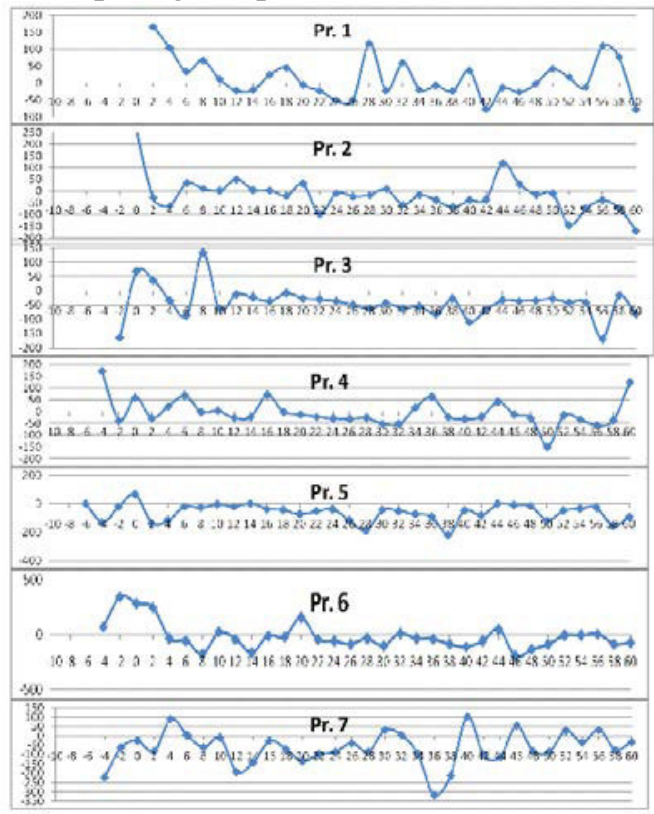

Fig. 2. Traitement primaire de la prospection magnétique effectuée (Correction sur les variations diurnes et le champ magnétique moyenne). L'axe horizontal - les piquets et l'axe vertical - champ corrigé en $n T$

\subsection{Traitement géologique des résultats de la prospection magnétique}

Pour trouver le caractère des anomalies magnétiques provoquées par des zones de broyage dans les dolérites nous avons construis le 
modèle géomagnétique (Fig. 3) et calculé l'anomalie magnétique d'après la formule ci-dessous:

$$
T=2 J \cdot 2 b \cdot(h \cos i+x \sin i) /\left(h^{2}+x^{2}\right)
$$

ici $T=$ module du vecteur totale du champ magnétique normale de la Terre $(0,25$ Oersted);

$J=$ vecteur d'aimantation (300 10-6 $\times 0,25$ Oersted);

$2 b=$ épaisseur horizontale de la zone de broyage $(20 \mathrm{~m})$;

$H=$ épaisseur de la couverture $(5 \mathrm{~m})$;

$I=$ angle d'inclinaison du vecteur $T(11 \mathrm{deg})$

$X=$ axe des abscisses;

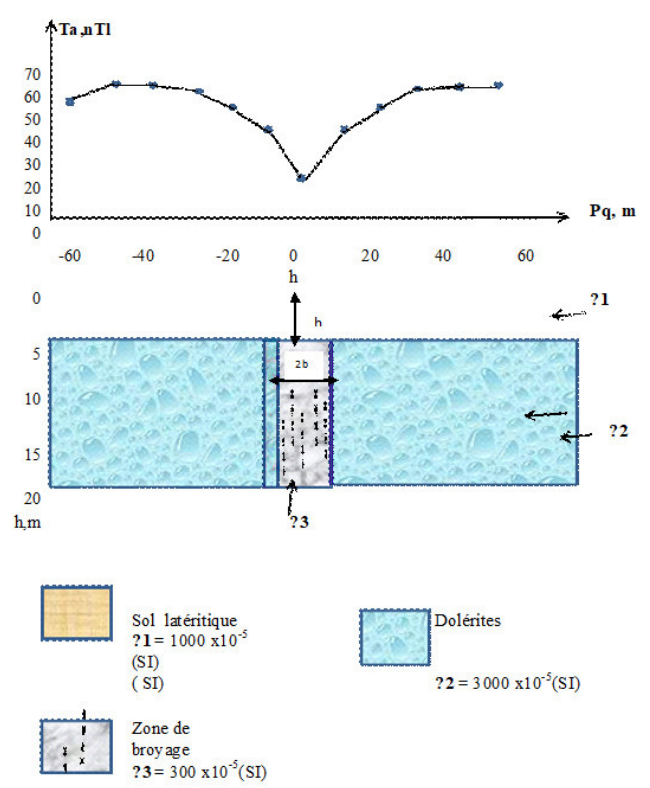

Fig.3. Anomalie magnétique provoquée par les zones de broyage

A partir de cette condition, on a trouvé que la valeur maximale d'anomalie à l'épicentre de la zone de broyage est égale à $6 \times 10^{-4}$ Oersted où $60 n T$.

Donc, la méthode de la prospection magnétique de haute précision, réalisée par le magnétomètre à proton, donne la possibilité à détecter les zones de broyage dans les coupes géologiques, qui sont favorables au point de vue alimentation en eau potable des villages. 


\section{Carte des profils de la prospection magnétiques}

Filtre $20 \times 20$ entre les profils et $30 \mathrm{~m}$ le longue des profils.

Le caractère des variations chaotiques du champ magnétique mesuré sur les profils (voir fig. 2), ne donne pas de possibilité effectuer correctement leur l'interprétation géologique puisque les anomalies d'origine géologique sont masquées par les variations apériodiques du champ magnétique d'origine industrielle.

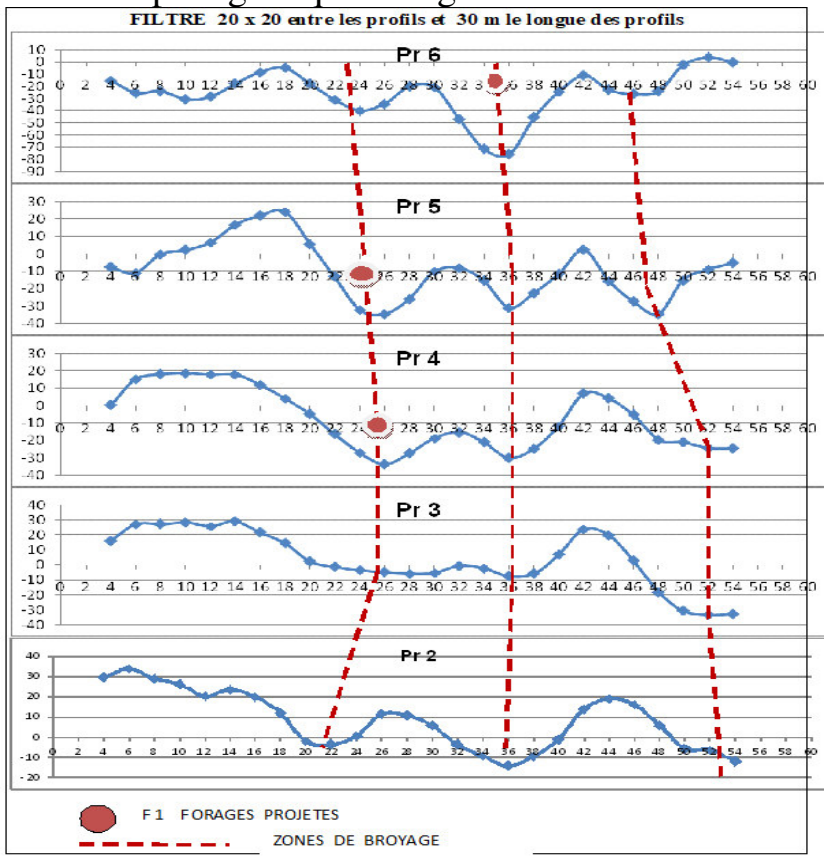

Fig.4. Traitement géologique de résultats de la prospection magnétique sur les sites

Pour diminuer l'influence des variations technogènes et démasquer les anomalies d'origine géologique, nous avons utilisé la filtration du champ magnétique mesuré par le filtre linéaire de $30 \mathrm{~m}$ de longue des profils et la fenêtre $20 \times 20 \mathrm{~m}$ entre les profils. Résultats de filtration sont représentés sur la fig.4.

Cette méthode de filtration a donné la possibilité découvrir les anomalies géologiques sur les profils d'une part et effectuer leur corrélation entre les profils d'autre part. 
Le caractère linéaire des anomalies donne la possibilité de les identifier comme des anomales provoqués par des zones de broyage et zones de fissures.

Sur la carte des profils (fig. 4) nous avons présenté les sites de trois forages hydrauliques implantés dans les zones de broyage.

\section{Conclusion}

La méthode magnétique, avec l'emploi d'un magnétomètre moderne à protons MMP-203MS, a été utilisée par TOUMNYNE sur les sites miniers de GBG de façon professionnelle.

Cette méthode a permis de détecter des fissures et des zones broyées sur les sites miniers qui constituent des structures favorables à l'accumulation des eaux souterraines et par conséquent des points favorables pour l'implantation des sondages d'adduction d'eau potable.

Il y a lieu de signaler dans cette zone minière, les variations apériodiques du champ magnétique d'origine industrielle qui provoquent variations chaotiques des valeurs sur les profils. C'est ainsi que la filtration des données recueillies sur le terrain a été utilisée pour éliminer ce phénomène en obtenant que des variations d'origine géologique pour le calcul des anomalies magnétiques.

Pour les travaux futurs dans cette région, nous recommandons l'utilisation de deux (02) stations de prospection magnétique, une fixe et l'autre mobile, pour éliminer les variations d'origine industrielle en évitant la filtration.

\section{Bibliographie}

1. Bundesanstalt für Geowissenchaften und Rohstoffe (BRG). La Géologie de la Chaîne des Bassarides et des Terrains environnants au Nord-Ouest de la Guinée, Hannover 2007 ;

2. Dr. Victorov Gleb. Propriétés physiques des roches, Boké 1992 ;

3. Dr. Victorov Gleb. Prospection géophysique, Conakry 1978 ;

Dr. Maraèv Igor. Géostatistique en géologie et dans les mines, Boké 2004 ;

4. Ph. Leblanc, Structure et hydrodynamique des milieux fissurés aquifères, Orléans 1986 ;

5. Wendgouda Priva KABRE, Caractérisation hydrogéologique d'un aquifère en milieu de socle fracturé : Cas De la province du Kourwéogo, Wagadougou 2012,

6. F. Radstake et Y. Chery, Prospection géophysique pour la recherche de l'eau souterraine en Haiti

7. Zéli Bruno Digbehi. Utilisation de la Prospection Géophysique par Résistivité Électrique Pour la Recherche d'eau Souterraine Dans le Département de TANDA (Est de la Cote D'IVOIRE).2002. 